\section{FRI0401 IMPLEMENTATION OF NICE GUIDELINES FOR OSTEOARTHRITIS IN PRIMARY CARE. FEASIBILITY STUDY OF JIGSAW-E IN SCOTLAND}

H. Frost ${ }^{1}$, J. Cowie ${ }^{2}$, T. Tooman ${ }^{3}$, K. Dziedzic ${ }^{4} .{ }^{1}$ Edinburgh Napier University, Sighthill Campus, Edinburgh, United Kingdom; ${ }^{2}$ Glasgow Caledonian University, Glasgow, United Kingdom; ${ }^{3}$ University of Dundee, Dundee, United Kingdom; ${ }^{4}$ Keele University, Keele, United Kingdom

Background: In the UK osteoarthritis $(\mathrm{OA})$ is a common musculoskeletal problem with 8.75 million people seeking treatment in $2015^{1}$. Evidence-based guidelines are available for the management of $\mathrm{OA}$ but implementation into routine daily practice remains complex. The Joint Implementation of Guidelines for Osteoarthritis in Western Europe (JIGSAW-E) model of care was developed and evaluated in England and implemented in Europe with an aim to optimise quality primary care for OA, support self-management and promote use of NICE guidelines ${ }^{2}$. The intervention includes:

1. An OA guidebook for patients

2. A model OA consultation for primary care

3. Training for practitioners to deliver the model consultation

4. Measures of quality care using an e-template

Objectives: To explore the feasibility of implementing the JIGSAW-E model to support people with OA in Scottish primary care.

Research questions were informed by the Theoretical Domains Framework with an aim of:

1) Exploring knowledge and beliefs about $\mathrm{OA}$ and its management in primary care.

2) Identifying determinants for change; barriers and facilitators to implementing the JIGSAW-E model in Scotland.

Methods: This qualitative study was comprised of 2 phases:

Phase 1 included semi-structured interviews with health professionals (GPs and Extended Scope Practitioners) working in primary care. A purposeful sampling approach aimed to provide geographical and professional representation across Scotland. Interviews were recorded, transcribed and analysed using a theoretically-informed thematic framework approach.

Phase 2 involved an engagement workshop that allowed for refinement and direct validation of emergent findings.

Results: 90 invitations were sent to practice managers in primary care. 14 participants from 10 practices across 6 Health Boards in Scotland were recruited for interviews, including 6 GPs and 8 Physiotherapy Extended Scope Practitioners (ESPs). 23 participants attended the engagement workshop $(E S P s=22$, GPs =1). Thematic analysis indicated four main themes related to the research questions:

1) Most participants were aware of NICE guidelines and believed they provided evidence-based OA care, and yet, for example, prescribing of co-codamol remained high. Physiotherapy ESPs were more likely to follow OA guidelines than GPs.

2) Adaptations of the JIGSAW-E model are needed to support OA management in the Scottish context. For example, in addition to adapting the guidebook for local relevance, the e-template was met with resistance due to technological barriers.

3) System-based barriers to implementation of the JIGSAW-E model included; lack of overall time for external training for practitioners; limited time in GP/ patient appointments to consult and explain medication use and importance of physical activity. In part because patients usually present with multi-morbidities.

4) The roll out of ESPs across Scotland in primary care provides a potential key for the delivery of sustainable evidence-based care in the Scottish health system.

Conclusion: Overall, participants were in favour of the JIGSAW-E model in Scotland. Contextual adaptation of written materials would increase acceptance, ownership and usability by both practitioners and patients. The evolving role of GPs and ESPs is key to implementation, where ESPs provide leadership in the delivery of evidence-based care for patients with osteoarthritis.

References:

[1] Arthritis Research UK. State of Musculoskeletal Health 2017.

[2] Dziedzic KS et al. Implementation of musculoskeletal Models of Care in primary care settings: Theory, practice, evaluation and outcomes for musculoskeletal health in high-income economies. Best practice \& research Clinical rheumatology 2016;30(3):375-97.

Acknowledgements: Thanks are due to the JIGSAW-E team, all participants, SISCC and EIT for funding.

Disclosure of Interests: None declared

DOI: 10.1136/annrheumdis-2020-eular.1239

\section{FRI0402 \\ MAGNETIC RESONANCE IMAGING EVALUATION OF CARTILAGE EROSION AND LIGAMENT INTEGRITY IN EARLY STAGE THUMB CARPOMETACARPAL JOINT OSTEOARTHRITIS}

H. S. Gong' ${ }^{1}$ K. J. Bae ${ }^{2}{ }^{1}$ Seoul National University Bundang Hospital, Orthopedic Surgery, Seongnam-si, Korea, Rep. of (South Korea); ${ }^{2}$ SMG-SNU Boramae Medical Center, Orthopedic Surgery, Seoul, Korea, Rep. of (South Korea)

Background: Most of the studies on pathogenesis of thumb carpometacaral joint (CMCJ-1) osteoarthritis were from cadavers or patients with advanced osteoarthritis, therefore the findings may not reflect any early changes of cartilage wear and ligament condition.

Objectives: We evaluated MRI to address where articular degeneration begins and which ligaments are most often involved in the early clinical stage CMCJ-1 osteoarthritis.

Methods: We retrospectively analyzed MRI examinations of 26 patients with early clinical stage CMCJ-1 osteoarthritis without radiologic abnormality and 19 control patients without CMCJ-1 pain or osteoarthritis who underwent MRI for dorsal or ulnar wrist pain. Two independent and blind observers assessed chondral defect of the CMCJ-1 divided into four quadrants: volar-ulnar (VU), volar-radial (VR), dorso-ulnar (DU), and dorso-radial (DR). They assessed the integrity of the four major ligaments of CMCJ-1: the anterior oblique ligament (AOL), the intermetacarpal ligament (IML), the posterior oblique ligament (POL), and the dorsal radial ligament (DRL). The prevalence of cartilage lesion and ligament abnormality between the osteoarthritic and control patients was compared using Fisher's exact test.

Results: Cartilage lesion was significantly more common in the VU quadrant of the trapezium in the osteoarthritic patients than in the control patients $(17 / 26$ vs. $2 / 19 ; P=0.002)$. AOL abnormality was more common in the osteoarthritic patients than in the control patients $(14 / 26$ vs. $3 / 19 ; P=0.009)$. In the osteoarthritic patients, 10 of 17 patients with $\mathrm{VU}$ quadrant cartilage erosion had $\mathrm{AOL}$ rupture, while four of nine patients without $\mathrm{VU}$ cartilage erosion had $\mathrm{AOL}$ rupture, thus there was no association between $\mathrm{VU}$ quadrant erosion and $\mathrm{AOL}$ rupture $(10 / 17$ vs $4 / 9, P=0.484)$

Conclusion: MRI evaluation of early clinical CMCJ-1 osteoarthritis commonly demonstrate cartilage lesion in the VU quadrant of the trapezium and ligament abnormality in the AOL. However, no association of cartilage erosion in the VU region and $A O L$ rupture suggests that $A O L$ rupture is not a mechanical factor leading to TMCJ osteoarthritis in specific area, but a common finding secondary to arthritic changes.

References:

[1] Ladd AL, Lee J, Hagert E. Macroscopic and microscopic analysis of the thumb carpometacarpal ligaments: a cadaveric study of ligament anatomy and histology. J Bone Joint Surg Am. 2012; 94(16):1468-1477.

[2] Williams A, Shetty SK, Burstein D, Day CS, McKenzie C. Delayed gadolinium enhanced MRI of cartilage (dGEMRIC) of the first carpometacarpal (1CMC) joint: a feasibility study. Osteoarthritis Cartilage. 2008; 16(4):530-532.

[3] Saltzherr MS, Coert JH, Selles RW, van Neck JW, Jaquet JB, van Osch GJ, et al. Accuracy of magnetic resonance imaging to detect cartilage loss in severe osteoarthritis of the first carpometacarpal joint: comparison with histological evaluation. Arthritis Res Ther. 2017; 19(1):55

[4] Dumont C, Lerzer S, Vafa MA, Tezval M, Dechent P, Sturmer KM, et al. Osteoarthritis of the carpometacarpal joint of the thumb: a new MR imaging technique for the standardized detection of relevant ligamental lesions. Skeletal Radiol. 2014; 43(10):1411-1420.

Disclosure of Interests: Hyun Sik Gong Speakers bureau: Amgen. Pfizer, Kee Jeong Bae: None declared

DOI: 10.1136/annrheumdis-2020-eular.878

\section{FRI0403 \\ CLINICAL FEATURES OF PROSTHETIC JOINT INFECTIONS DIFFER IN PATIENTS WITH INFLAMMATORY ARTHRITIS AND OSTEOARTHRITIS}

M. Kapadia ${ }^{1}$, T. Pannellini ${ }^{1}$, C. Moezinia ${ }^{1}$, A. Miller ${ }^{1}$, M. Figgie ${ }^{1}$, P. Sculco ${ }^{1}$, M. Cross ${ }^{1}$, M. Henry ${ }^{1}$, L. Russell', L. Donlin ${ }^{1}$, A. Nocon ${ }^{1}$, S. Goodman ${ }^{1}$. ${ }^{1}$ Hospital for Special Surgery, New York, United States of America

Background: Inflammatory arthritis (IA) patients are at increased risk for prosthetic joint infections (PJI). However, because active IA patients without infections can have elevated inflammatory markers that mimic joint infection, PJI diagnosis is challenging in this population.

Objectives: We used an institutional PJI registry to identify and compare the clinical, microbiologic, and histopathologic features of culture positive (CP) and culture negative (CN) total hip and knee PJI in IA and OA patients. We 
also evaluated the relationship between culture positivity, IA, and clinical outcomes.

Methods: A retrospective cohort of THA/TKA PJIs, from 2009 to 2016, were identified by ICD codes, and confirmed by chart review. IA diagnosis was also confirmed by use of IA-specific medications. CN cases were defined as PJIs with no evidence of microbial growth in intraoperative cultures and CP PJI cases were defined by positive microbial growth in intraoperative cultures. Treatment failure was defined as subsequent surgical treatment for infection after the initial infection surgery. H\&E slides of OA and IA PJI cases matched by age (+/-5) sex, and culture status were reviewed by a pathologist for evidence of the histopathologic features listed in Table 2. Fisher's exact test, chi-square test, and Kaplan-Meier estimates were used.

Results: 807 PJI cases were identified including 36 IA (33 RA and 3 SLE) and 771 OA. A higher proportion of IA PJI were CN $(N=10,27 \%)$ vs. OA PJI ( $N=109$, $14 \%, p=0.02)$. IA-PJI were younger, female, on glucocorticoids, and with more comorbidities. Type of surgical treatment did not differ significantly between IA and OA groups. Comparing CN-IA vs. CP-IA, no difference was observed in age, smoking, diabetes, surgical treatment, IA-specific meds or Charlson comorbidities. One-year survivorship of $\mathrm{CN}-\mathrm{IA}$ and $\mathrm{CN}-\mathrm{OA}$ were $66 \%$ and $87 \%$ ( $p>0.05)$. Across all CP cases, $57 \%$ were staphylococcal, with no differences between groups. Treatment failure was more frequent for CP-IA (42\%) compared to CP-OA $(30 \%),(p=0.2)$

Histopathology of 88 PJIs (31 IA and 57 OA) was reviewed. The IA cohort presented with more chronic inflammation $(p=0.001)$ than the OA cohort. Within the IA cohort, a higher proportion of CP-IA had $>10 P M N$ per HPF $(p=0.003)$ and met MSIS criteria $(p=0.009)$. Comparing CP-OA and $\mathrm{CN}-\mathrm{OA}$, there were no significant differences in histopathology findings or number of patients meeting MSIS criteria.

Conclusion: IA PJls are more likely to be culture negative than OA PJIs. Although our analysis was limited by our cohort size, our findings including differences in histopathology, and better clinical outcomes suggest the presence of biologic differences between $\mathrm{CN}$ and $\mathrm{CP} P \mathrm{PI}$ that require further study.

TABLE 1. Patient characteristics in IA and OA PJIs

\begin{tabular}{|c|c|c|c|c|c|}
\hline & \multicolumn{2}{|c|}{ IA } & \multicolumn{2}{|c|}{ OA } & \multirow[b]{2}{*}{$\mathrm{p}$-value } \\
\hline & $\mathbf{N}$ & $\% / S D$ & $\mathrm{~N}$ & $\% / S D$ & \\
\hline Total & 36 & & 771 & & \\
\hline Age & 58.5 & 11.4 & 66.8 & 12 & $<.001$ \\
\hline BMI & 30.2 & 6.7 & 30 & 6.7 & 0.861 \\
\hline Female & 28 & 77.8 & 332 & 43.1 & $<.001$ \\
\hline $\mathrm{CCl}$ & 2.8 & 1.7 & 1.7 & 2.1 & 0.002 \\
\hline Smoking & 4 & 11.1 & 86 & 11.2 & 0.792 \\
\hline Glucorticoids & 10 & 27.8 & 39 & 5.1 & $<.001$ \\
\hline Culture Negative & 10 & 27.8 & 109 & 14.1 & 0.024 \\
\hline Treatment Success at 2 years & 19 & 52.8 & 509 & 66 & 0.146 \\
\hline
\end{tabular}

IA- inflammatory arthritis; OA - osteoarthritis; PJI -prosthetic joint infection; CCI - Charlson Comorbidity Index

TABLE 2. Histopathology and clinical presentation in IA and OA PJIs

\begin{tabular}{|c|c|c|c|c|c|c|}
\hline & $\mathrm{OA}(\mathrm{N}=57)$ & $\mathrm{IA}(\mathrm{N}=31)$ & \multirow[b]{2}{*}{$\mathrm{p}$-value } & \multirow{2}{*}{$\frac{C P-I A(N=23)}{N(\%)}$} & \multirow[t]{2}{*}{$\mathrm{CN}-\mathrm{IA}(\mathrm{N}=8)$} & \multirow[b]{2}{*}{$\mathrm{p}$-value } \\
\hline & \multicolumn{2}{|l|}{$N(\%)$} & & & & \\
\hline \multicolumn{7}{|l|}{ Pathology Review } \\
\hline >10 PMN per HPF & $42(74)$ & $22(71)$ & 0.806 & $20(87)$ & $2(25)$ & 0.003 \\
\hline Chronic Inflammation & $13(23)$ & $23(74)$ & 0.001 & $18(78)$ & $5(63)$ & 0.393 \\
\hline Necrosis & $17(30)$ & $9(29)$ & 1 & $8(35)$ & $1(13)$ & 0.38 \\
\hline \multicolumn{7}{|l|}{ Clinical Presentation } \\
\hline MSIS & $50(88)$ & $26(84)$ & 0.747 & $22(96)$ & $4(50)$ & 0.009 \\
\hline Sinus Tract & 7 (12) & 7 (23) & 0.233 & $5(22)$ & $2(25)$ & 1 \\
\hline Elevated ESR or CRP & $41(72)$ & $24(77)$ & 0.622 & $17(74)$ & 7 (88) & 1 \\
\hline Elevated Synovial WBC & $33(58)$ & $19(61)$ & 0.823 & $13(57)$ & $6(75)$ & 1 \\
\hline Elevated Synovial \%PMN & $31(54)$ & $20(65)$ & 0.377 & $14(61)$ & $6(75)$ & 0.333 \\
\hline
\end{tabular}

OA - osteoarthritis; IA - inflammatory arthritis; CP - culture positive; CN - culture negative; MSIS - meets Musculoskeletal Infection Society diagnostic criteria

Disclosure of Interests: Milan Kapadia: None declared, Tania Pannellini: None declared, Carine Moezinia: None declared, Andy Miller: None declared, Mark Figgie: None declared, Peter Sculco: None declared, Michael Cross: None declared, Michael Henry: None declared, Linda Russell: None declared, Laura Donlin Consultant of: Consultant - Genentech/Roche, Allina Nocon: None declared, Susan Goodman Shareholder of: Reginosine- Investment, Grant/research support from: Novartis, Horizon, Consultant of: Novartis, Celgene, UCB

DOI: 10.1136/annrheumdis-2020-eular.4777

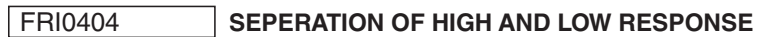 GROUPS IN OSTEOARTHRITIS USING SERUM CARTILAGE DEGRADATION AND FORMATION MARKERS - A 3 YEAR FOLLOW-UP ON THE FORWARD STUDY TESTING THE EFFICACY OF SPRIFERMIN}

H. Gühring ${ }^{1}$, A. C. Bay-Jensen ${ }^{2}$, F. Moreau' ${ }^{1}$, J. R. Andersen ${ }^{3}$, A. R. Bihlet ${ }^{3}$, M. Karsdal ${ }^{2} .{ }^{1}$ Merck KGaA, Darmstadt, Germany; ${ }^{2}$ Nordic Bioscience, Immunoscience, Herlev, Denmark; ${ }^{3}$ Nordic Bioscience, Clinical development, Herlev, Denmark

Background: In osteoarthritis (OA), response to different interventions could be more pronounced in different endotypes of cartilage turnover. Data from UK biobank suggest that an endotype of low cartilage repair is associated with structural progression in osteoarthritis $(\mathrm{OA})[1]$. Sprifermin a truncated and recombinant FGF18 have been shown to induce chondrocyte proliferation and cartilage formation in in vitro settings[2-4]. Efficacy on cartilage thickness in OA was recently demonstrated in the FORWARD study[5].

Objectives: We investigated markers of cartilage formation (serum PRO-C2) and degradation (urine CTX-II), to assess profiles indicative of chondrocyte metabolic activity would be associated with both structural and symptomatic responses to sprifermin.

Methods: Serum and urine from participants of the FORWARD study, a phase II clinical trial testing the efficacy of intra-articular (IA) sprifermin, were collected throughout the study. Clinical data recorded at baseline, year 2 and year 3 follow-up were used, including cartilage thickness and WOMAC. All available baseline samples of the placebo and treatment arms were assessed for serum PRO-C2 and urinary CTX-II. Patients were separated into dichotomized groups based on 33,50 or 66 percentiles cut-points and two-year treatment responses were compared in a prospective-retrospective statistical design manner.

Results: Patients with low baseline PRO-C2 $(<66 \%)$, in the $100 \mathrm{ug} / 4 \mathrm{x}$ treatment arm, had greater difference to placebo in cartilage thickness as compared to the high PRO-C2 and all-comers groups ( 0.06 vs 0.03 and $0.05 \mathrm{~mm}$, respectively) at two and three years (Fig.). Similar effect was seen for low CTX-II (<50\%) $(0.07$ vs -0.03 and $0.05 \mathrm{~mm}$, respectively). In addition, the WOMAC placebo effect was reduced in the low PRO-C2 and the low CTX-II groups, resulting in a difference compared to placebo of -0.81 vs. 1.35 (low vs high proC2 and -2.35 vs 1.47 (low vs high CTX-II).

Conclusion: We noticed that low baseline PRO-C2 and CTX-II indicative for low metabolic activity of chondrocytes were associated with improved symptomatic outcome and slightly increased cartilage thickness compared to high metabolic activity. The parallelism between PRO-C2 and CTX-II point towards the existence of a low cartilage repair endotype and might reflect a subgroup of patients with higher sensitivity towards interventions - an effect that was maintained over three years.

Fig. 1: Longitudinal change in cartilage thickness $(\mathrm{mm})$ in the total femorotibial joint in patients with low and high levels of PRO-C2 (top panel) and CTX-II (lower panel). Data are shown as mean with 95\% $\mathrm{Cl}$ of absolute change from baseline up to 3 Years.
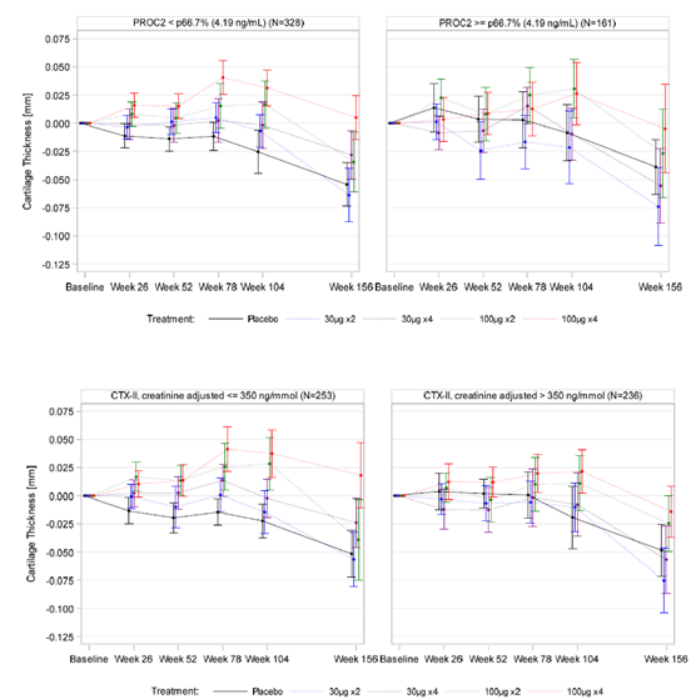